\title{
The complex long-term X-ray activity of the compact binary 4U 1915-05`
}

\begin{abstract}
V. Šimon
Astronomical Institute, Academy of Sciences of the Czech Republic, 25165 Ondřejov, Czech Republic

e-mail: simon@asu.cas.cz

Received 25 August 2004 / Accepted 17 January 2005

Abstract. An analysis of the long-term X-ray activity of the compact binary 4U 1915-05 from 1996-2003, using the $A S M / R X T E$ data, shows that the behaviour of the long-term X-ray light curve inside this interval was considerably different from that reported previously. We find that the X-ray light curve is dominated by complicated variations in the form of outbursts with rather sharp peaks, each lasting for about 20-30 days. The period search by weighted wavelet transform (WWZ) reveals that the cycle-length of the activity is highly variable and the periods can persist only through several epochs. We find that a cycle of about 230-250 days (similar to the $\sim 199$ day cycle reported by Priedhorsky \& Terrell 1984, ApJ, 280, 661) occurred only in a limited time interval - this cycle manifests in the variations of the recurrence time and amplitude of the outbursts. Generally, the system spends more time in the low state than in the high state (outbursts), and the standard deviation and skewness display variations on the time scale of the order of hundreds of days. We show that the decays of the individual outbursts in 4U $1915-05$ display a relatively broad range of slopes. The slow average decay rate $\tau_{\mathrm{D}} \approx 0.2 \mathrm{ct} / \mathrm{s} /$ day can be related to the viscous time of the disk. We discuss the models and offer an interpretation in terms of the thermal instability of the disk, modified by irradiation by the neutron star, following the model by King \& Ritter (1998, MNRAS, 293, L42). We also compare the activity of 4 U 1915-05 with that of a similar system, 4U 1820-30, and interpret the largely divergent kinds of activity in terms of the different mean mass transfer rates. This rate is lower in 4U 1915-05 and can allow thermal instability of the disk, unlike the case of 4U 1820-30. We argue that the disk in 4U 1915-05 can oscillate between thermally stable and unstable state.
\end{abstract}

Key words. stars: neutron - stars: binaries: close - circumstellar matter - X-rays: binaries - stars: individual: 4U 1915-05

\section{Introduction}

4U 1915-05 is an X-ray binary with an ultra-short orbital period $P_{\text {orb }}=50 \mathrm{~min}$ (Walter et al. 1982). It therefore belongs to the group of compact binaries, i.e. systems that consist of two compact objects. The donor component is a partly degenerate star because $P_{\text {orb }}$ is too short to accommodate a non-degenerate star (Nelson et al. 1986; Pylyser \& Savonije 1988, 1989). The mass-gaining object is a neutron star (NS) because it undergoes X-ray bursts (Becker et al. 1977).

The X-ray orbital modulation of 4U 1915-05 displays dips of a largely variable width and depth that sometimes disappear completely (Chou et al. 2001). On the other hand, the optical modulation has a rather smooth profile and a period longer by about 1 percent than the X-ray period (e.g. Chou et al. 2001). The 1 percent difference between these periods is highly significant because the X-ray dip period is measured to be $3000.6508 \pm 0.0009 \mathrm{~s}$ while the optical modulation period is $3027.5510 \pm 0.0052 \mathrm{~s}$; in addition, there is a beat period of $\sim 3.9$ day, manifesting in the modulation of the X-ray dip shape (Chou et al. 2001). According to the latest interpretation, 4U 1915-05 possesses both positive and negative

* This research has made use of the observations provided by the $A S M / R X T E$ team. superhumps caused by disk precession as in some cataclysmic variables (Retter et al. 2002), and the triple-star model (Grindlay et al. 1988) is not necessary.

4U 1915-05 displays a strong long-term X-ray activity but its periodicity is yet unclear. A cycle of 199 days, with possible superimposed 40 day variations, was suggested by Priedhorsky \& Terrell (1984), who used the 10-day means of VELA 5B observations. Later, Smale \& Lochner (1992) reanalyzed the longer cycle in the same data and argued that it is not very strong, with a confidence level of just about 80 percent. Homer et al. (2001) barely detected the possible periods of 8.6, 12, 23 and 83 days in the first four years of the ASM/RXTE observations by the PDM (Stellingwerf 1978) and Lomb-Scargle methods (Lomb 1976; Scargle 1982). 4U 1915-05 is classified as an atoll source that displays a similar path in the X-ray color-color diagram as another compact binary, 4U 1820-30, in spite of its systematically significantly lower luminosity (Bloser et al. 2000). The atoll path in the X-ray color-color diagram implies that the long-term activity is caused by a variable accretion of matter onto the NS, and not by disk precession.

In this paper we analyze the character of the long-term activity of 4U 1915-05 using the data from the All Sky Monitor (ASM) onboard the Rossi X-ray Timing Explorer (RXTE) which provides data of an unprecedented quality for this object 

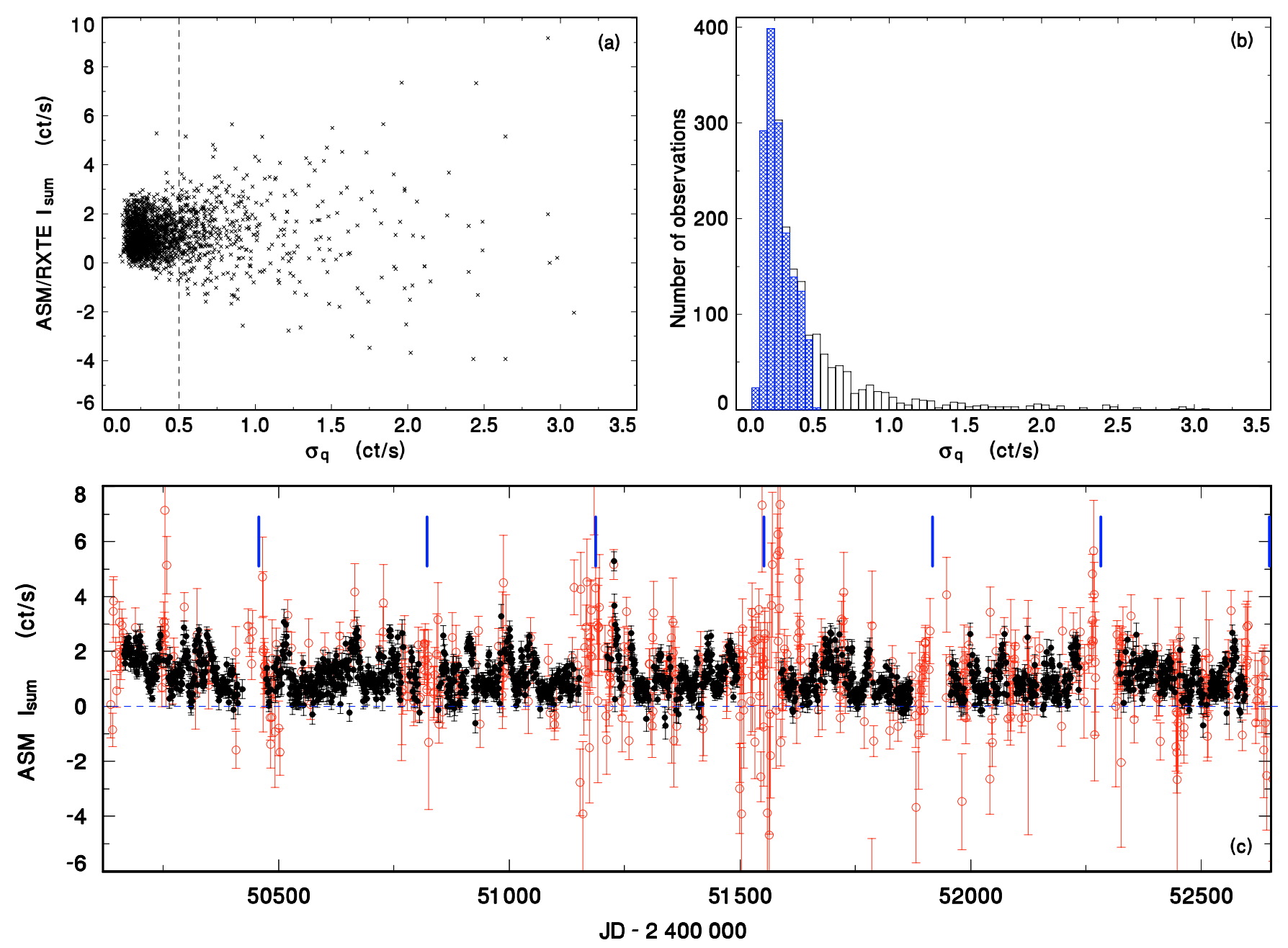

Fig. 1. a) $I_{\text {sum }}$ of all the original ASM/RXTE one-day means (1.5-12 keV) of $4 \mathrm{U} 1915-05$ within the years 1996-2003, plotted versus the quoted uncertainty $\sigma_{\mathrm{q}}$. The vertical dashed line represents $\sigma_{\mathrm{q}}=0.5 \mathrm{ct} / \mathrm{s}$. Notice that there is no dependence of $\sigma_{\mathrm{q}}$ on $I_{\text {sum. }}$. A few points fall out of the range of the plot. b) Histogram of $\sigma_{\mathrm{q}}$ of the data set. The observations with $\sigma_{\mathrm{q}} \leq 0.5 \mathrm{ct} / \mathrm{s}$ are marked by the filled bars. c) The X-ray light curve over the years 1996-2003. The filled and empty circles denote the measurements with the quoted $\sigma_{\mathrm{q}} \leq 0.5 \mathrm{ct} / \mathrm{s}$ and $\sigma_{\mathrm{q}}>0.5 \mathrm{ct} / \mathrm{s}$, respectively. Notice how much the light curve improves when the most noisy data are omitted. The vertical lines mark the moments of the conjunction of 4U 1915-05 with the Sun. See Sect. 3.1 for details. (This figure is available in color in electronic form.)

and enables us to analyze fine features. In Sect. 3.1 we analyze the morphology of the X-ray variations and show that the behaviour of the system appears to differ from that reported previously. We investigate its time behaviour by various methods in Sect. 3.2, including an analysis of the statistical properties of the intensity variations. A search for the periodicity, carried out using a weighted wavelet Z-transform, is presented in Sect. 3.3. An analysis of the properties of the individual outbursts, particularly their decaying branches, is performed in Sect. 3.3.1. An interpretation of the activity is given in Sect. 4, including a comparison with a similar system, 4U 1820-30. A preliminary version of a part of this analysis was presented by Šmon (2004b).

\section{Source of the data}

The activity of 4U 1915-05 from 1996-2003 was observed by ASM/RXTE (Levine et al. 1996) (http://xte.mit.edu/). Only one-day means of the sum band intensities $I_{\text {sum }}$
(1.5-12 keV) were used for our purposes in order to increase the signal/noise ratio. This binning is sufficient because our analysis concentrates on the long-term activity and hence on the features in the light curve on the time scale of days and longer. Modified Julian Date, used by RXTE, was transformed into the more often used Julian Date. Hereafter, it is used in the form of JD - 2400000 .

\section{Data analysis}

\subsection{General description}

The signal of $4 \mathrm{U} 1915-05$ detected by ASM/RXTE is often relatively weak. In order to assess the quality of the data, $I_{\text {sum }}$ of all the original one-day means was plotted versus the quoted uncertainty $\sigma_{\mathrm{q}}$ (Fig. 1a). It can be readily seen that most points cluster in a small region of the diagram, with outliers towards larger $\sigma_{\mathrm{q}}$. There is no dependence of $\sigma_{\mathrm{q}}$ on $I_{\text {sum. }}$. The histogram for $\sigma_{\mathrm{q}}$ of the data set is shown in Fig. 1b. The trials showed 

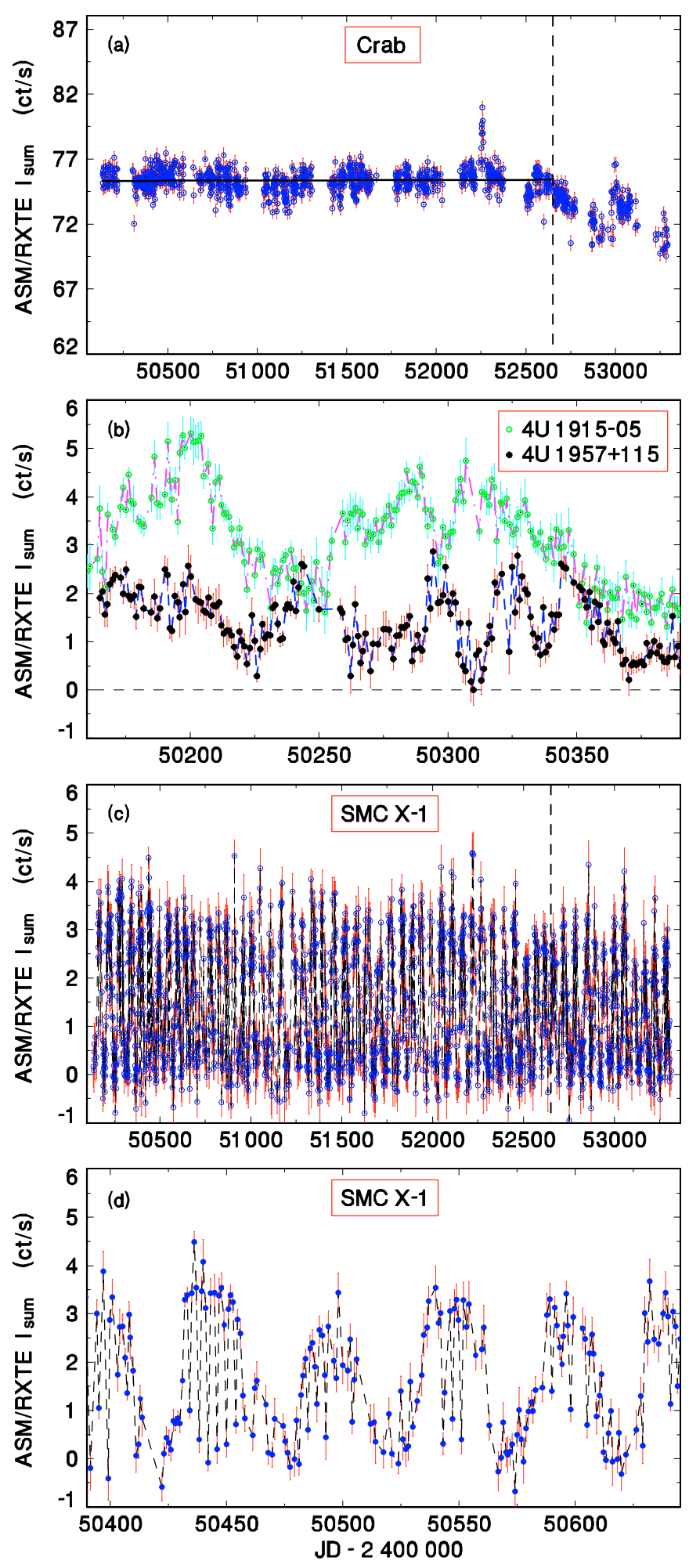

Fig. 2. The $I_{\text {sum }}$ light curves (one-day means; $1.5-12 \mathrm{keV}$ ) documenting the performance of $A S M / R X T E$. a) The light curve of the Crab pulsar, containing the data with $\sigma_{\mathrm{q}} \leq 0.6 \mathrm{ct} / \mathrm{s}$. b) A comparison of the light curves of 4U 1915-05 and 4U 1957+115. c) The whole light curve of SMC X-1. d) A segment of the light curve of SMC X-1, documenting the reliability of the profile even for a faint source; the narrow fadings are due to the eclipses. Only the data with $\sigma_{\mathrm{q}} \leq 0.5 \mathrm{ct} / \mathrm{s}$ were used in b), c) and d). See Sect. 3.1 for details. (This figure is available in color in electronic form.) that dividing the measurements according to their $\sigma_{\mathrm{q}}$ to the groups with $\sigma_{\mathrm{q}} \leq 0.5 \mathrm{ct} / \mathrm{s}$ and $\sigma_{\mathrm{q}}>0.5 \mathrm{ct} / \mathrm{s}$ considerably suppressed the noise and largely improved the appearance of the profile of the light curve (Fig. 1c). At the same time, the curve containing only the data with $\sigma_{\mathrm{q}} \leq 0.5 \mathrm{ct} / \mathrm{s}$ remained dense enough to resolve its profile, with only a few intervals of missing data, mostly due to the conjunctions with the Sun. Only the daily means with $\sigma_{\mathrm{q}} \leq 0.5 \mathrm{ct} / \mathrm{s}$ were therefore used for further analysis.

Since the performance of $A S M / R X T E$ is subject to changes over time, and since 4U $1915-05$ is a rather faint object for this instrument, a careful check of the reliability of the features of the light curve was made using comparison with other sources. Figure 2a shows the light curve of the Crab pulsar, here containing the data with $\sigma_{\mathrm{q}} \leq 0.6 \mathrm{ct} / \mathrm{s}$. It can be seen that the part of the curve to the left of the vertical dashed line can be well fitted by a straight line with zero slope - this indicates good calibration and stability of $A S M$. Waves and a decrease of $I_{\text {sum }}$ appeared after JD 2452650; this interval therefore was not used for our analysis of 4U 1915-05.

A comparison of the light curve of $4 \mathrm{U} 1915-05$ with those of two similarly faint sources was made. The criterion described above, i.e. the restriction to the data with $\sigma_{\mathrm{q}} \leq 0.5 \mathrm{ct} / \mathrm{s}$, was applied here. Figure $2 \mathrm{~b}$ shows a comparison of the light curves of $4 \mathrm{U} 1915-05$ and $4 \mathrm{U} 1957+115$. It can be readily seen that the long-term $A S M$ light curves are well defined for both sources, with quite independent features. Another example, including the whole light curve of SMC X-1, is presented in Fig. 2c. A segment of this curve in Fig. 2d documents the reliability of the profile of the cyclic long-term variability even for such a faint source. Notice the narrow fadings which are due to the eclipses on its 3.892 day orbital period (e.g. Schreier et al. 1972; Tuohy \& Rapley 1975).

The light curve of 4U 1915-05 plotted on an expanded time scale can be seen in Fig. 3 (only the measurements with $\sigma \leq 0.5 \mathrm{ct} / \mathrm{s}$ ). $I_{\text {sum }}$ varies between about 0.3 and $3 \mathrm{ct} / \mathrm{s}$. The signal is therefore rather weak but a careful fitting of the curves by several methods carried out here can reveal the features on the curve with very good confidence. As regards the confidence with which the features on the $A S M / R X T E X$-ray light curve can be taken, in addition to the tests described above, Leahy (2002) was able to obtain reliable $A S M$ curves for GX 301-2 which spends a large part of the time at $I_{\text {sum }} \approx 0.5-2 \mathrm{ct} / \mathrm{s}$, away from the vicinity of phase $\sim 0.9$ of its 41.491 day orbit, that is mostly at a similar countrate as $4 \mathrm{U} 1915-05$. The $A S M$ intensity of LMC X-3, analysed by Boyd \& Smale (2004), varies between 0 and $4 \mathrm{ct} / \mathrm{s}$, with mean count rate of $1.64 \mathrm{ct} / \mathrm{s}$. McGowan et al. (2004) studied long-term X-ray variations during non-periodic outbursts of SS Cyg, whose AS M signal is even fainter, typically $0.5-1 \mathrm{ct} / \mathrm{s}$.

We note that the X-ray modulation (dips) does not appear to affect the profile of the long-term light curve of 4U 1915-05 significantly. The reason is that the daily means of $I_{\text {sum }}$ were usually determined from 10-30 measurements spread over time.

The X-ray light curve in Fig. 3 represents about 12 epochs of the 199 day cycle reported by Priedhorsky \& Terrell (1984). As can be seen from Fig. 3, the profile of the light curve is 

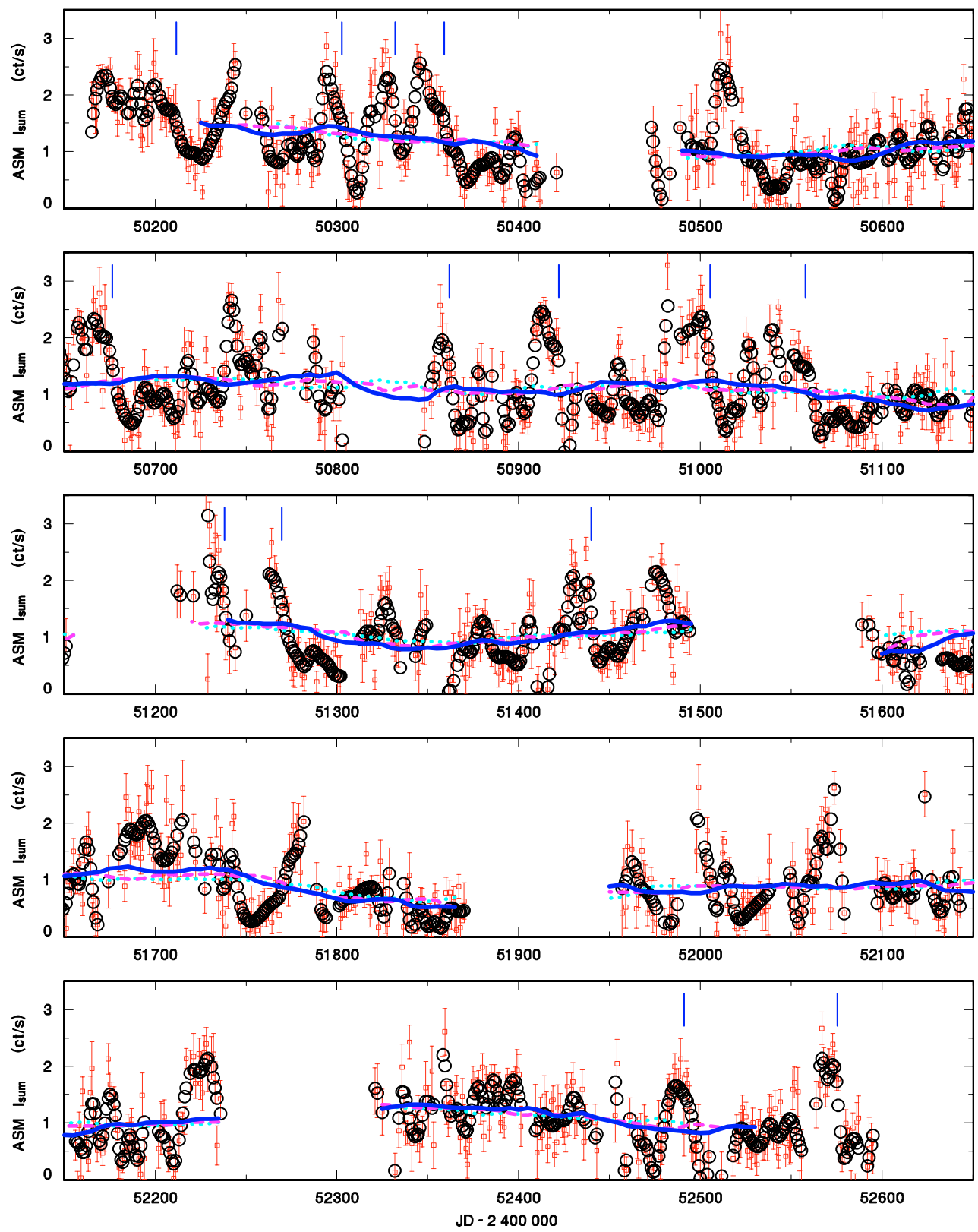

Fig. 3. The ASM/RXTE X-ray light curve of $4 \mathrm{U} 1915-05$. Only the one-day means of $I_{\text {sum }}$ with $\sigma_{\mathrm{q}} \leq 0.5 \mathrm{ct} / \mathrm{s}$ are plotted (empty squares with error bars corresponding to $\sigma_{\mathrm{q}}$ ). In addition, the isolated points in seasonal gaps were removed. The empty circles represent the fit by the code HEC13. The thick lines denote the fits to the HEC13 points by the moving averages for $Q=60$ (solid line), 80 (dashed line), 100 days (dotted line). The arrows mark the well covered decay branches analyzed in Sect. 3.3.1 and displayed in Fig. 10. (This figure is available in color in electronic form.)

very complicated, often with appreciable variations, even on short time scales of several days and weeks. In order to clearly show the smooth profile of the long-term variations, the curve was fitted by the code HEC13, written by Dr. P. Harmanec. The code is based on the method of Vondrák $(1969,1977)$, who improved the original method of Whittaker (Whittaker \& Robinson 1946). The method is based on minimizing the value $Q=F+\lambda^{2} S$, where $F$ denotes the fidelity of the graduated to the ungraduated values, $S$ is the smoothness of the graduated curve, and $\lambda^{2}$ is a constant defining the degree of graduation. This method can fit a smooth curve to the non-equidistant data no matter what their profile is. HEC13 makes use of two input parameters, $\epsilon$ and $\Delta T . \epsilon=1 / \lambda^{2}$ determines how "tight" the fit will be, that is if only the main profile or also the highfrequency variations are to be reproduced. $\Delta T$ is the interval over which the data are binned before smoothing. The resulting fit consists of the mean points, calculated to the individual observed points of the curve. After several trials, the fit with $\epsilon=10^{-1}, \Delta T=4$ days was found to satisfy the general profile of the curve. The smoothed curve is plotted by the large open circles in Fig. 3. 


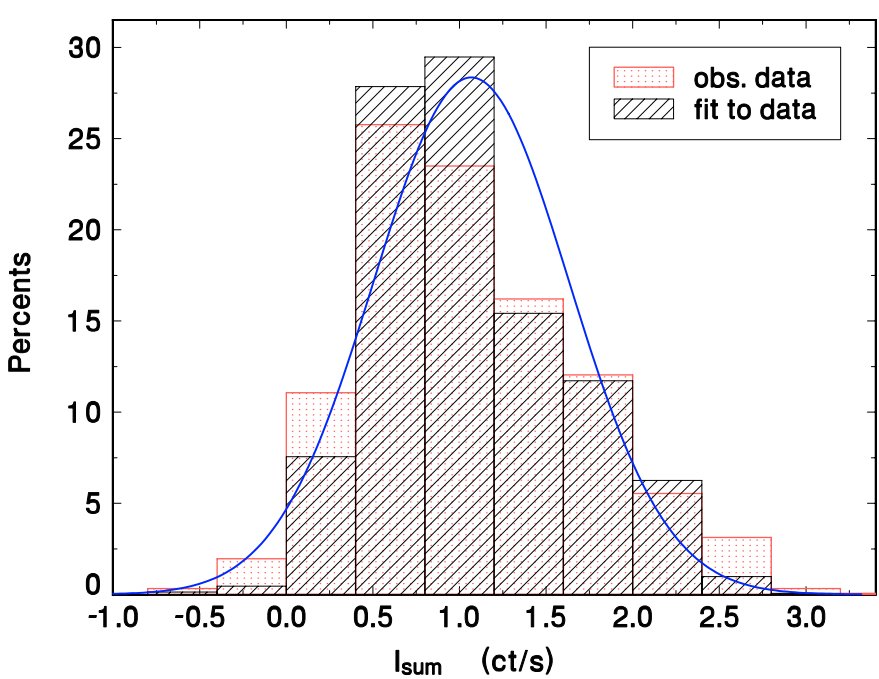

Fig. 4. The statistical distribution of $I_{\text {sum }}$ of $4 \mathrm{U}$ 1915-05. The histograms for the observed data and for the fit by HEC13 from Fig. 3 are displayed, with binning of $0.4 \mathrm{ct} / \mathrm{s}$. The Gaussian curve for the fit by HEC13 is plotted for comparison. See Sect. 3.1 for details. (This figure is available in color in electronic form.)

The statistical distribution of $I_{\text {sum }}$ for the observed data from Fig. 3 is displayed in Fig. 4. It can be seen that it differs from Gaussian, with a pronounced asymmetry between the left-hand and right-hand side of the histogram. The slope of the right-hand side appears to be significantly less steep.

\subsection{Long-term evolution of the activity}

In principle, the cycle of about 200 days reported by the previous analyses can be realized through a variable recurrence time and amplitude of the outbursts, and thus it may not be easily resolvable. The X-ray light curve was therefore heavily averaged to pick up the variations on the long time scale. The curves, calculated by HEC13 in the previous step, were smoothed by the two-sided moving averages with the filter halfwidth $Q=60,80$ and 100 days, in steps of 5 days. $Q$ refers to the semi-interval within which the data were averaged. The results are shown in detail in Fig. 3. It can be readily seen that the individual fits are in good agreement with each other. The condensed time scale in Fig. 5a enables a better assessment of the profile of the long-term changes.

The moving averages also enabled us to calculate the temporal variations of the standard deviation $\sigma$ and skewness $\gamma_{1}$ of the X-ray light curve, and to obtain thus the measures of the variations of the $I_{\text {sum }}$ curve on the time scale of months and years. Both $\sigma$ and $\gamma_{1}$ were calculated from both the HEC13 curves and from the residuals of the moving averages of the HEC13 curve. The resulting curves are displayed in Fig. 5b,c and show that there are no large differences between the curves determined for the HEC13 fits directly and for the residuals. Figure $5 \mathrm{c}$ shows the smoothed profile of $\gamma_{1} \cdot \gamma_{1}=0$ corresponds to the symmetric distribution of $I_{\text {sum }}$ with respect to the mean. $\gamma_{1}>0$ and $\gamma_{1}<0$ correspond to the asymmetric distributions with a tail towards more positive or negative values, respectively. It can be seen from Fig. $5 \mathrm{c}$ that $\gamma_{1}>0$ dominates.

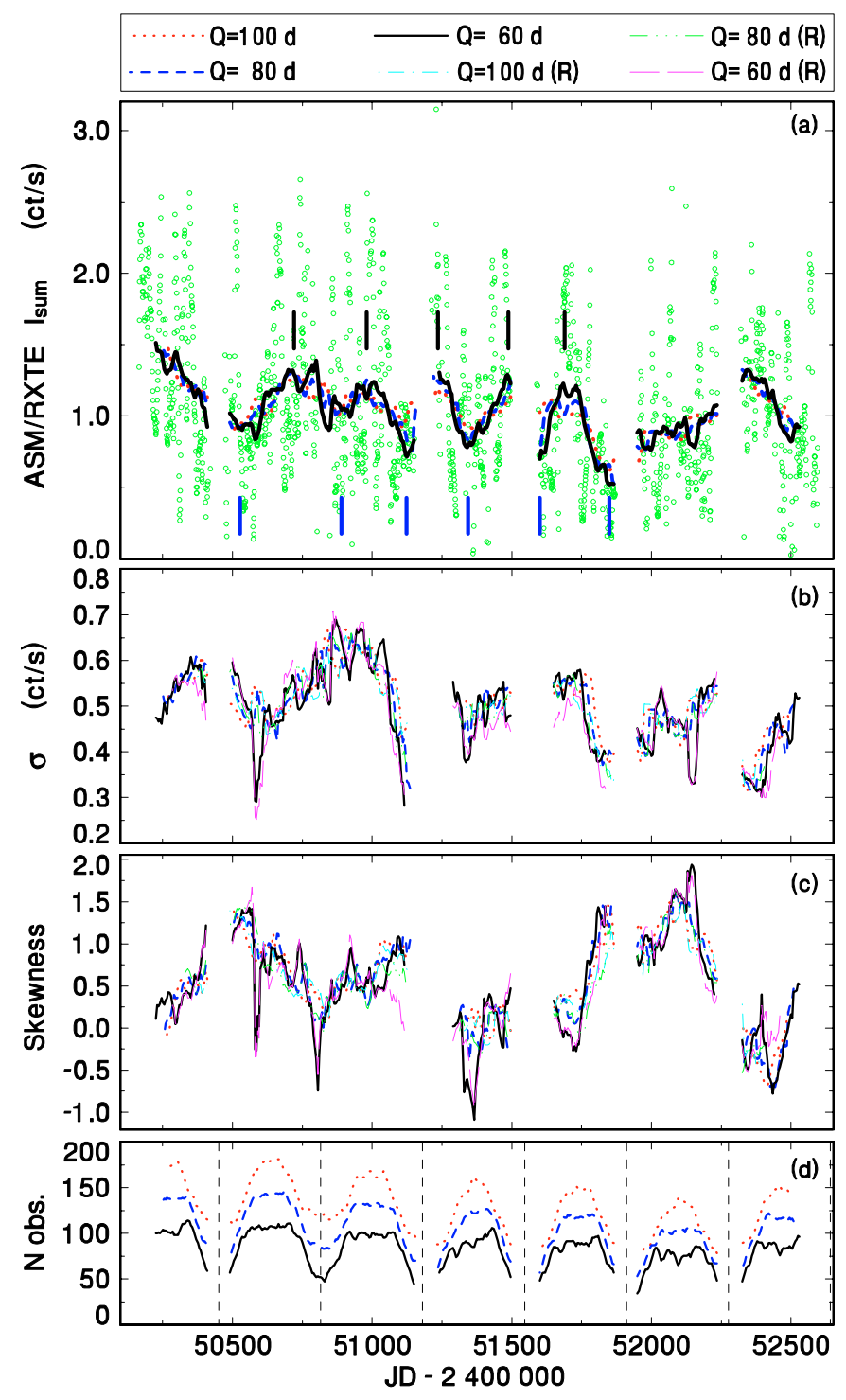

Fig. 5. a) The X-ray light curve of $4 \mathrm{U} 1915-05$ on condensed time scale. The curves calculated by HEC13 (empty circles; from Fig. 3) and their moving averages (thick lines) are displayed. The maxima and minima of the cycle of about 250 days are marked. b) The profile of the standard deviation $\sigma$. c) The profile of the skewness $\gamma_{1}$. d) The number of observations entering the moving averages. The values of $Q$ (in days) are included in the box; $\mathrm{R}$ denotes that $\sigma$ and $\gamma_{1}$ were determined for the residuals of the moving averages. See Sect. 3.1 for details. (This figure is available in color in electronic form.)

Figure 5a,b,c shows that 4U 1915-05 displays variations of the parameters of activity on a very long time scale, of the order of almost a thousand days.

Figure 5 shows that neither $\sigma$ nor $\gamma_{1}$ appear to be related to the profile of the moving averages of $I_{\text {sum }}$. On the other hand, there is an indication of a dependence between $\sigma$ and $\gamma_{1} . \sigma$ was therefore plotted versus $\gamma_{1}$ (Fig. 6). In order to lower the scatter, the curves for all values of $Q$ (both the HEC13 curves and their residuals of the moving averages) were merged and binned into 5 day means. It can be readily seen that although the relation between $\sigma$ and $\gamma_{1}$ is complicated and undergoes long-term variations, the curves occupy only some locations in 


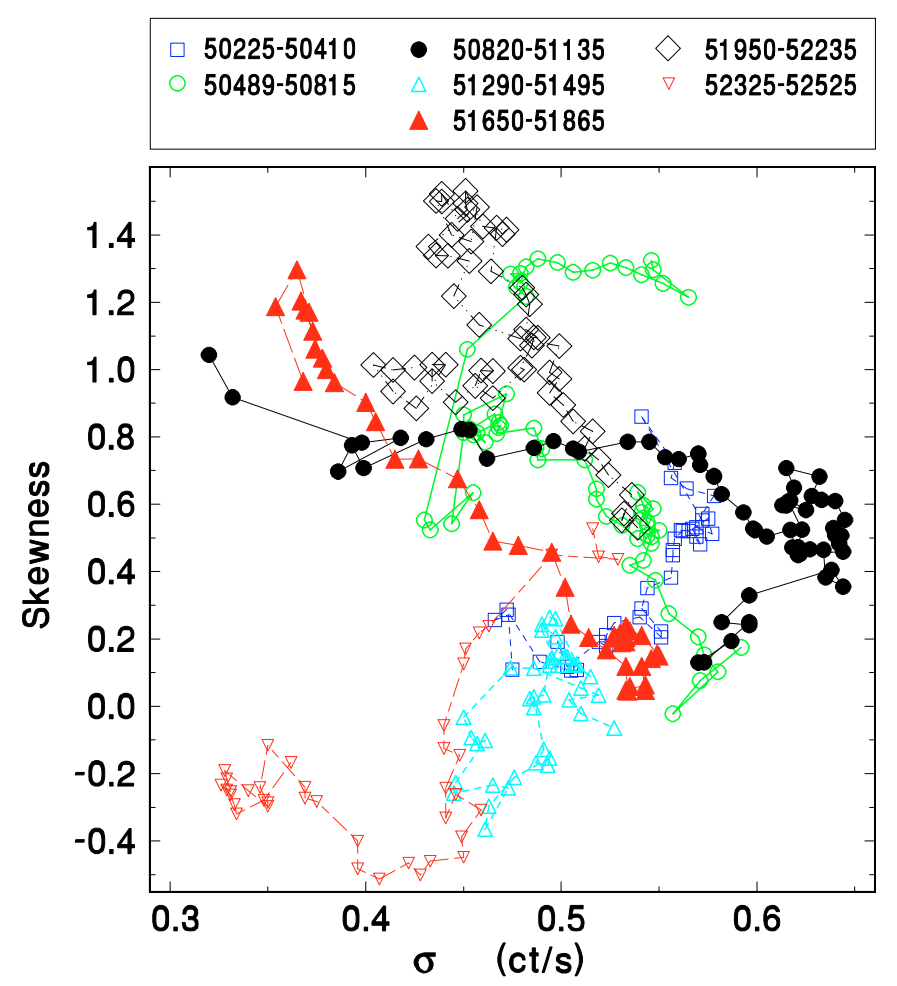

Fig. 6. The relation between $\sigma$ and $\gamma_{1}$ from Fig. 5b,c. The line connecting the points illustrates the time evolution; for convenience, the observations in the individual years, marked in Fig. 5d, are plotted with different symbols. The legend lists the time intervals of JD - 2400000 . See Sect. 3.2 for details. (This figure is available in color in electronic form.)

the diagram. An anticorrelation dominates although a positive correlation is apparent in some time intervals.

In order to better understand Fig. 6, we simulate the relation between $\sigma$ and $\gamma_{1}$ in Fig. 7. We assume here that the amplitude of $I_{\text {sum }}$ remains constant and only the time fraction spent in the high state increases through points No. 1 to $14 . \sigma$ grows until the symmetrical distribution of $I_{\text {sum }}$ is reached $\left(\gamma_{1}=0\right)$, and decreases later on when the system begins to spend more time in the high state than in the low state. It can be seen that the basic pattern of variations from Fig. 6 can be plausibly reproduced.

\subsection{The cycle lengths}

A search for the cycle lengths in the ASM/RXTE data set was carried out using a weighted wavelet Z-transform (WWZ) (Foster 1996; wWw.aavso.org/data/software/ wwz.shtml). This method, making use of the Morlet wavelet, enables one to determine the period and amplitude of unevenly sampled time series and is thus very suitable for discovering possible temporarily existing periods in the activity of $4 \mathrm{U}$ 1915-05. Here the method of wavelets is used for the first time for the analysis of the time behaviour of 4U 1915-05. It will be shown that it yields very important information about the complicated appearance and disappearance of the cycles in this system.

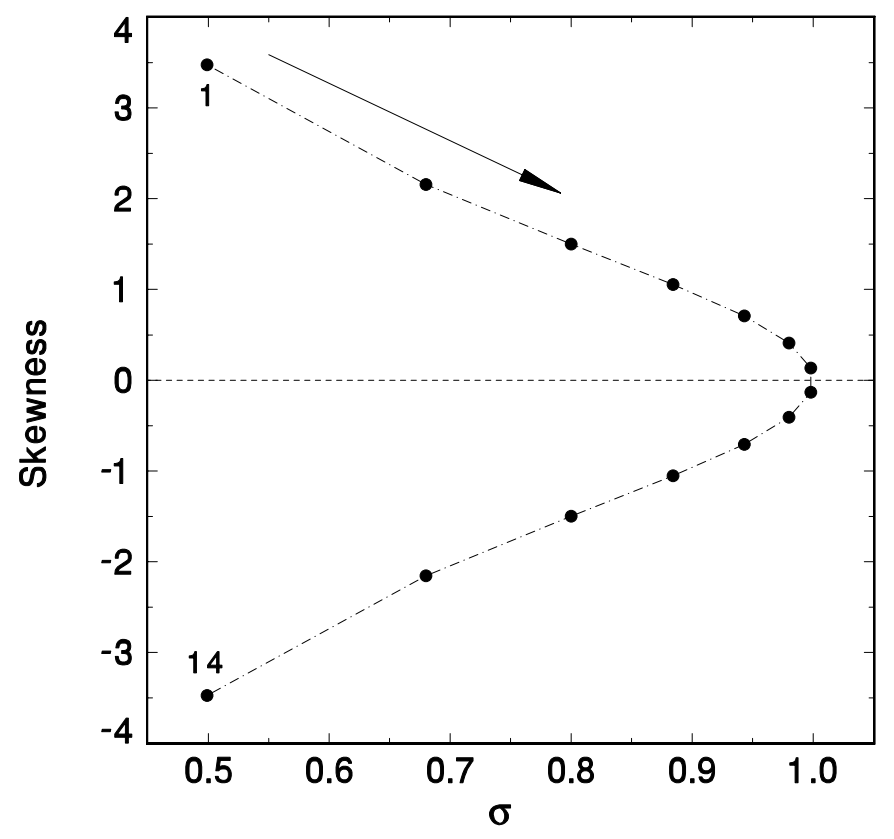

Fig. 7. Simulation of the relation between $\sigma$ and $\gamma_{1}$ for a constant amplitude of $I_{\text {sum }}$ (the units are arbitrary). The horizontal dashed line divides the regions of negative and positive correlation between $\sigma$ and $\gamma_{1}$. The arrow shows the evolution with an increasing time fraction spent in the high state. See Sect. 3.2 for details.

WWZ indicates whether or not there is a periodic fluctuation at a given time at a given frequency. The value of the parameter $c$ which determines how rapidly the analyzing wavelet decays was set to 0.0125 , as recommended for the analyses of the variable star light curves by Foster (1996).

The result for the curve fitted by HEC13 is shown in Fig. 8. The upper limit of the displayed frequency range roughly corresponds to the duration of the outbursts in the X-ray light curve. It can be seen that the periods appear and disappear throughout the covered time interval and none of them persists all the time. The periods are often subject to large changes and multiperiodicity even appears to be present in some intervals.

In order to better examine the long periods, the light curve smoothed by $Q=60$ days was submitted to the WWZ transform, too. A much simpler diagram in comparison with Fig. 8 was obtained - it unambiguously displays only the period of $\sim 230$ days (frequency of about 0.0043 day $^{-1}$ ), already apparent in Fig. 8a,c between JD 2451 000-JD 2451 900. This cycle can even be resolved directly in the moving averages in Fig. 5a.

An O-C diagram could be constructed for the maxima and minima of the $\sim 230$ day cycle. It is shown in Fig. 9. The ephemeris for the mean cycle-length of 250 days yields an almost zero slope of the $\mathrm{O}-\mathrm{C}$ residuals, although real fluctuations are apparent. This period is in plausible agreement with the result of the wavelet analysis. The $\mathrm{O}-\mathrm{C}$ method enables us to analyze these variations between JD 2450500 and JD 2451900 , that is in a longer interval than possible with WWZ. 

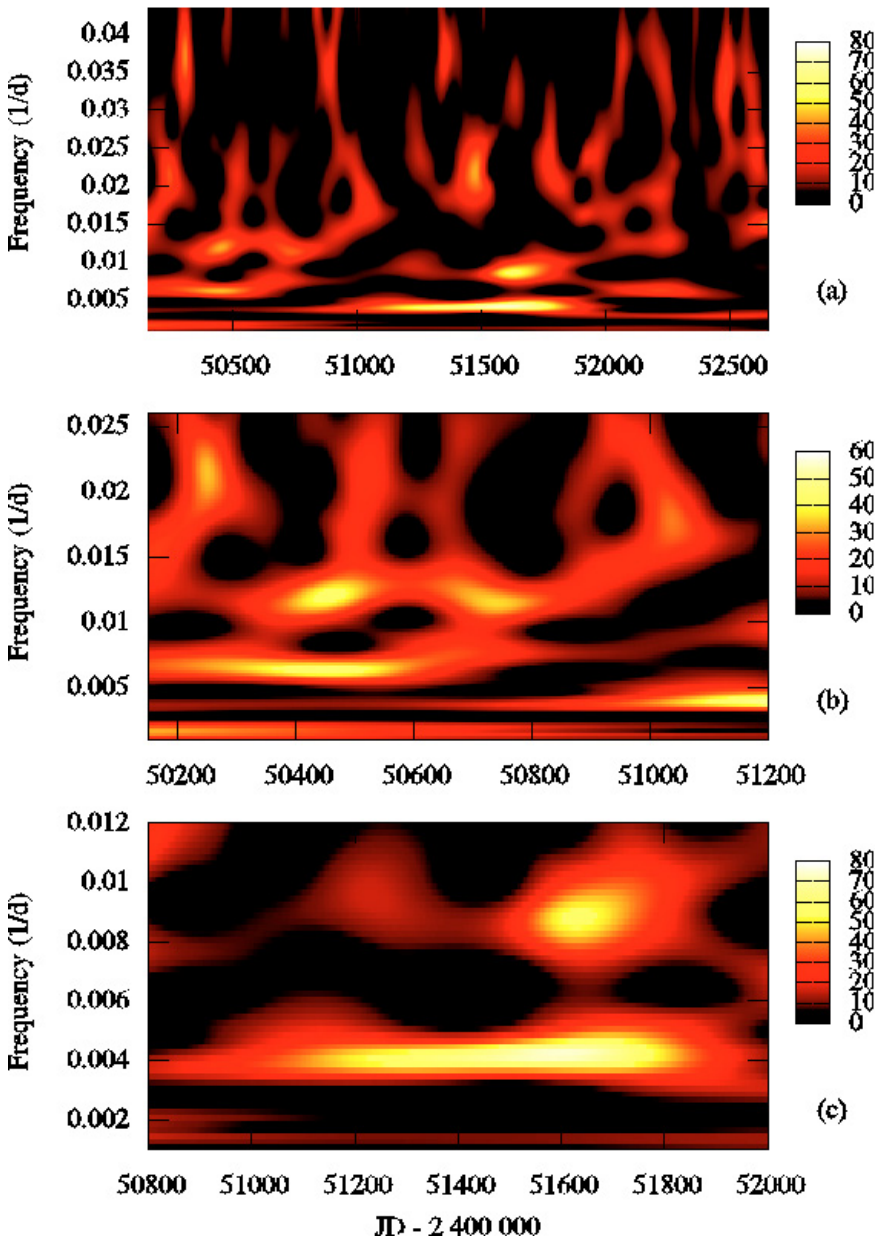

Fig. 8. Weighted wavelet Z-transform of the X-ray light curve from Fig. 3 for the fit by HEC13. The whole dataset is displayed in a) while a segment with a complicated evolution of the periods is shown in $\mathbf{b}$ ). The interval in which the $\sim 230$ day cycle occurred is plotted in c); the dotted curve indicates the maximum of WWZ. The color scale represents the values of WWZ. See Sect. 3.3 for details. (This figure is available in color in electronic form.)

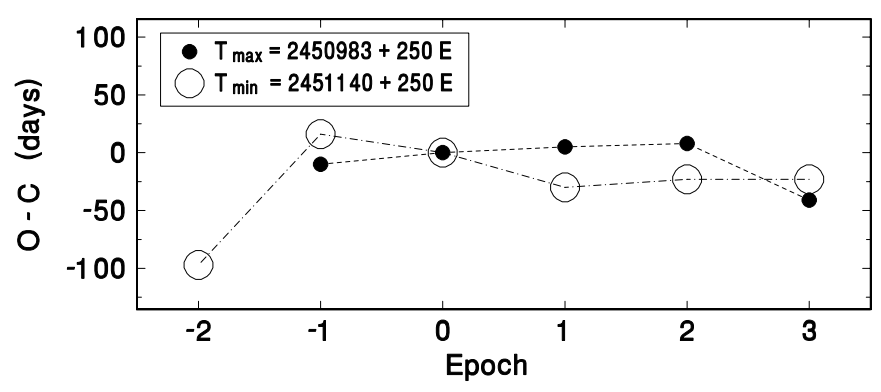

Fig. 9. The $\mathrm{O}-\mathrm{C}$ diagram for the maxima and minima of the moving averages from Fig. 5a between JD 2450500 and JD 2451900 . The length of the ordinate is equal to the length of the mean period of 250 days to allow for an assessment of the fluctuations.

\subsubsection{Decaying branches of outbursts}

The profile of the X-ray light curve of $4 \mathrm{U} 1915-05$ is quite complicated. Relatively rapid variations are common and usually have the form of outbursts with sharp peaks. Generally, a study of the morphology of the outbursts can best be made by
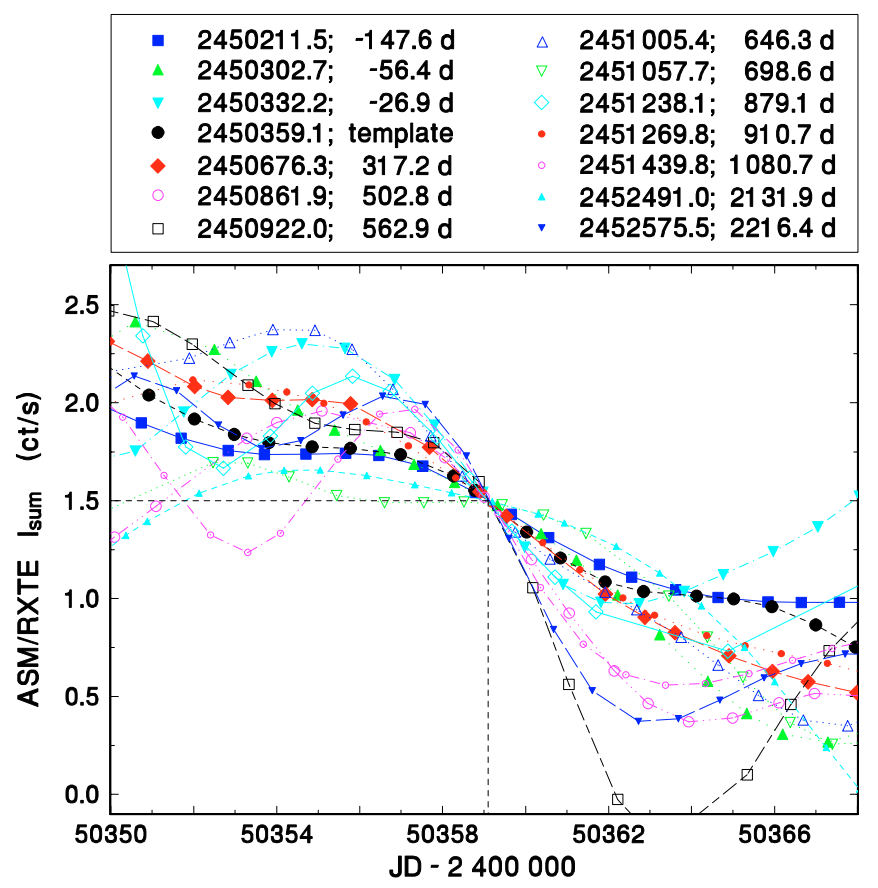

Fig. 10. Comparison of the profiles of the decaying branches of the outbursts of $4 \mathrm{U} 1915-05$. The profile fitted by HEC13 is shown for each event. The individual curves were shifted along the time axis to match the template - the time of crossing $I_{\text {sum }}=1.5 \mathrm{ct} / \mathrm{s}$ (in JD) and the shifts with respect to the template are listed in the figure. See Sect. 3.3.1 for details. (This figure is available in color in electronic form.)

matching the events to a representative outburst, taken as a template (e.g. Šimon 2002, 2004d). Since the range of the decay rates of the events in 4U 1915-05 was found to be larger than in soft X-ray transients (SXTs), the method had to be modified. $I_{\text {sum }}=1.5 \mathrm{ct} / \mathrm{s}$ was chosen as the reference level, in which the curve of a given event was matched to the template. The superposition of the decaying branches of the individual events then enables us to assess the range of their decay rates. The decaying branch of the event that occurred in JD 2450359 was chosen as the template. 14 observed events with good coverage and relatively smooth profile could be used (Fig. 10); they are also marked in Fig. 3. The typical duration of the decay $D$ with the amplitude of $1.6 \mathrm{ct} / \mathrm{s}$ is $\sim 8.2$ days, which yields the average decay rate $\tau_{\mathrm{D}} \approx 0.2 \mathrm{ct} / \mathrm{s} /$ day.

\section{Discussion}

Our analysis of the ASM/RXTE data of 4U 1915-05, spanning the years 1996-2003, reveals that the behaviour of the longterm X-ray light curve is different from that reported previously. We show that the dominant feature of activity is outbursts, whose typical duration is 20-30 days with a recurrence time $T_{\mathrm{C}} \approx 30-80$ days. These outbursts mostly possess sharp peaks without a plateau, and any long intervals of high state are very rare. The statistical distribution of $I_{\text {sum }}$ shows that 4U 1915-05 spends more time in the low state than in the high state, that is, in outburst. 
We find several cycle lengths $P$ in the X-ray light curve. However, they undergo large variations and, moreover, they appear and disappear during the covered time interval; e.g. one $P$ changed from $\sim 83$ days to 60 days in the interval of JD 2450360-JD 2451080. $P \approx 83$ days appears to correspond to that found by Homer et al. (2001) using PDM and Lomb-Scargle methods. Moreover, Fig. 8 reveals that this period was accompanied by a longer cycle of $\sim 155$ days until JD 2450 600; we also note that this period may be resolved in the PDM periodogram of Homer et al. (2001). We can see the time evolution and eventual disappearance of these periods. We argue that the shorter of these two periods ( $\sim 83$ days) can be attributed to the periodicity of $T_{\mathrm{C}}$ while the longer one cannot it is caused by the modulations of $T_{\mathrm{C}}$ on longer time scales. We note that these periods do not reappear in later epochs and we can conclude that any period shorter than about 200 days does not persist for more than about half a year (Fig. 8).

A very important finding is that we detected $P \approx$ 230-250 days by two independent methods. This cycle is similar to that of $\sim 200$ days, reported by Priedhorsky \& Terrell (1984) and Smale \& Lochner (1992), but it appears only for a limited time interval and displays a largely variable amplitude. This $P$ displays noticeable fluctuations and manifests as a variable $T_{\mathrm{C}}$ and amplitude of the outbursts.

We show that $\sigma$ and $\gamma_{1}$ of the X-ray light curve of 4U 191505 undergo appreciable variations on the time scale of the order of hundreds of days. These quantities are usually anticorrelated, although occasional intervals of a positive correlation can be resolved, too. This relation can be plausibly modeled by the dominant variations of the time fraction spent in outburst. The positive correlation occurs only when $I_{\text {sum }}$ has a rather symmetric distribution and its amplitude is suppressed. This correlation extends to a too high $\gamma_{1}$ than what can be explained purely by the changes of the time fraction spent in outburst, therefore also the variations of the amplitude of $I_{\text {sum }}$ and the quiescent level can play a role to some extent. The positive correlation can occur for both high and low mean $I_{\text {sum }}$ (compare Figs. 5 and 3). The first episode occurred when the initially high mean $I_{\text {sum }}$ was declining, with the onset of outbursts with almost no quiescent intervals. Another episode occurred when the system was fluctuating in the quiescent state with only faint outbursts. The variations of $\sigma$ and $\gamma_{1}$ are only weakly related to the cycle lengths. However, the large changes of $P$, when one cycle begins or finishes, can be reflected in the variations of $\sigma$. The episode of large and rapid decrease of $\sigma$ near JD 2451080 corresponds to the moment when a cycle of $\sim 60$ days finished and a cycle of $\sim 250$ days strengthened (Figs. 5 and 8 ). The disappearance of the $\sim 250$ day cycle was then accompanied by another large decrease of $\sigma$ near JD 2451850 . Such transitions between cycles thus appear to be accompanied by a suppression of outbursts.

In principle, processes such as variations of the mass transfer rate from the donor, thermal instability (TI) of the disk, disk precession, the Mazeh \& Shaham (1979) mechanism (a third body influencing the eccentricity of the close pair and thus modulating the mass outflow from the donor) can give rise to the variations in the X-ray intensity in X-ray binaries on time scales longer than $P_{\text {orb }}$. The periodicity can be more or less inherent in these mechanisms, but may not be always easily detectable. Boyd \& Smale (2004) revealed that the longterm activity of Cyg X-2, LMC X-3 and Cyg X-3 can be characterized in terms of integer multiples of some fundamental period, and in Cyg X-2 even coincident with the multiples of $P_{\text {orb }}$. The integer multiples manifest themselves as deep troughs of intensity and occur in a random order in a given system. This phenomenon arises from the combination of the periodic and chaotic component of the activity and may be common to several possible mechanisms. Boyd \& Smale (2004) proposed several explanations, incorporating geometrical effects (as in Her X-1, e.g. Gerend \& Boynton 1976), or variations of the magnetic fields in the disk. Since the intensity variations of LMC X-3 are known to be accompanied by spectral changes (Wilms et al. 2001), variable mass accretion is involved, at least in this system.

Although the character of the activity in 4U 1915-05 makes it difficult to decide if the phenomenon found by Boyd \& Smale (2004) is present also in this system, we can at least determine some constraints. The dominant form of the X-ray intensity variations are brightenings from the low state, which is a behaviour different to that of the systems analysed by Boyd \& Smale (2004). The quiescent level of $I_{\text {sum }}$ of 4U 1915-05 remains about the same and does not appear to display any occasional deep, well-defined troughs of $I_{\text {sum }}$ such as those observed in the systems analysed by Boyd \& Smale (2004). The $\sim 230-250$ day cycle arises from the variations of the properties of the outbursts. As we will argue below, these outbursts can be attributed to TI, hence the phenomenon would have to manifest as the modulation of the ionization conditions of the disk. It is not possible to determine if the $\sim 230-250$ day cycle is related to $P_{\text {orb }}$ in this system because their ratio would be extremely high. The $\sim 230-250$ day cycle repeats about five times in our data set, albeit with some fluctuations - this would suggest that only a single integer multiple (or a set of closely spaced multiples) of a fundamental period was operating in this case. The difference in the kind of activity of 4U 1915-05 from the systems in Boyd \& Smale's (2004) study may lie in the relatively low X-ray luminosity of this binary (see below).

Disk precession is not an acceptable explanation for 4U 1915-05 because, although chaotic behaviour is possible for a specific set of parameters, this system lies in the parameter space where the precession is impossible (Wijers \& Pringle 1999). Moreover, we note that $4 \mathrm{U} 1915-05$ moved along the atoll path in the X-ray color-color diagram during the outbursts (Bloser et al. 2000). This suggests that the outbursts are really a result of a variable accretion of matter onto the NS, and not the effects of disk precession, which would imply an occultation and/or absorption effect.

The properties of the activity of 4U 1915-05 suggest that the influence of a third body via the Mazeh \& Shaham (1979) mechanism (Grindlay et al. 1988) is not the dominant mechanism driving the observed activity. We conclude that any longterm cycle in 4 U 1915-05 is much less stable in comparison with another ultracompact atoll system, 4U 1820-30, in which this mechanism can operate (Chou \& Grindlay 2001; Šimon 2003), and the behaviour of both systems is in striking variance with each other. It thus turns out that although both 
4U 1915-05 and 4U 1820-30 are ultracompact atoll sources, they display largely divergent long-term X-ray activity, specific for each system. The causes of variation of the mass accretion rate onto the NS, $\dot{m}_{\mathrm{NS}}$, therefore must be largely different in each system.

We argue that the large difference between the luminosity at minimum of $4 \mathrm{U} 1915-05$ and $4 \mathrm{U} 1820-30\left(L_{\mathrm{X}}=3 \times\right.$ $10^{36} \mathrm{erg} \mathrm{s}^{-1}$ (Barret et al. 1996) versus $L_{\mathrm{X}}=2 \times 10^{37} \mathrm{erg} \mathrm{s}^{-1}$, Stella et al. 1987), and a larger disk radius in 4U 1915-05 can play an important role in their largely divergent X-ray activity and can serve as a guide to the investigation of the physical mechanisms. The radius of the disk in 4U 1915-05 is $1.45 \times 10^{10} \mathrm{~cm}$ for $R_{\text {disk }} \approx 0.9 R_{\text {lobe }}$ (the disk is expected to fill a large part of the lobe because superhumps are detected). Applying the models for $\mathrm{He}, \mathrm{C}$, and $\mathrm{O}$ disks (Menou et al. 2002), we obtain the critical mass transfer rate $\dot{m}_{\text {crit }}$ below which TI can occur to be $1.16 \times 10^{17} \mathrm{~g} \mathrm{~s}^{-1}$ (He disk), $2.32 \times 10^{16} \mathrm{~g} \mathrm{~s}^{-1}(\mathrm{C}), 8.52 \times 10^{16} \mathrm{~g} \mathrm{~s}^{-1}(\mathrm{O})$, and $2.13 \times 10^{16} \mathrm{~g} \mathrm{~s}^{-1}$ $(\mathrm{C} / \mathrm{O})$. The observed X-ray luminosity of $4 \mathrm{U} 1915-05$ at minimum implies $\dot{m}_{\min } \approx 1.6 \times 10^{16} \mathrm{~g} \mathrm{~s}^{-1}\left(2.5 \times 10^{-10} M_{\odot} \mathrm{yr}^{-1}\right)$, assuming a $1.4 M_{\odot}$ and $10 \mathrm{~km}$ radius NS, which is slightly lower than $\dot{m}_{\text {crit }}$ - this suggests that the disk can be thermally unstable, and this can contribute to the observed activity. The short $T_{\mathrm{C}}$ and a large fraction of time spent in outburst indicate that the disk balances between the thermally stable and unstable state; the interval prior to JD 2450400 when the decline of the mean $I_{\text {sum }}$ was accompanied by the onset of the outbursts with almost no time spent in quiescence is of particular importance in this respect. The observed amplitude of the X-ray outbursts of 4U 1915-05 implies that the mass accretion rate onto the NS decays by only $\sim 80$ percent within $\sim 5-15$ days, which is much less than in SXTs. The amplitude of the optical variations on the time scale of days, comparable to the time scale of the X-ray outbursts, is smaller than that of $I_{\text {sum }}$ $(\Delta V \approx 0.5 \mathrm{mag}$; Callanan et al. 1995). This can be explained by the longer decay time scale of the optical radiation due to the spectral shift to longer wavelengths (King \& Ritter 1998; Chevalier \& Ilovaisky 1995).

The observed optical luminosity of 4U 1915-05 provides us with evidence for the irradiation of the disk by the NS. In the case of purely viscous heating of the disk, $\dot{m}_{\min } \approx$ $2.5 \times 10^{-10} M_{\odot} \mathrm{yr}^{-1}$ at minimum X-ray luminosity would yield the observed magnitude $m_{\mathrm{V}} \approx 23.2$ for the distance of $9.3 \mathrm{kpc}$ (Yoshida 1993), inclination angle $i \approx 70^{\circ}$ (appropriate for the dipping LMXBs, van Paradijs et al. 1988), interstellar extinction $A_{\mathrm{V}}=1.35 \mathrm{mag}$ (from $N_{\mathrm{H}}=2 \times 10^{21} \mathrm{~cm}^{-2}$, Bloser et al. 2000) and using the $A_{\mathrm{V}}-N_{\mathrm{H}}$ relation by Predehl \& Schmitt (1995). This is fainter than the observed $m_{\mathrm{V}} \approx 21$ (Grindlay et al. 1988). Increasing $\dot{m}$ to about $10^{-9} M_{\odot} \mathrm{yr}^{-1}$ in the outburst peak would still give $m_{\mathrm{V}} \approx 22.5$. We note that although this irradiation increases the effective disk temperature, the observed positive and negative superhumps imply that the disk is asymmetric (see the models by Truss et al. 2001) and tilted (Retter et al. 2002). The irradiation is thus non-uniform and some parts of the disk can be even shielded at a given time. In addition, a disk structure like spiral arms (Truss et al. 2002) can play a role in shielding of the outer disk region - a part of the disk can thus remain below the hydrogen ionization temperature.

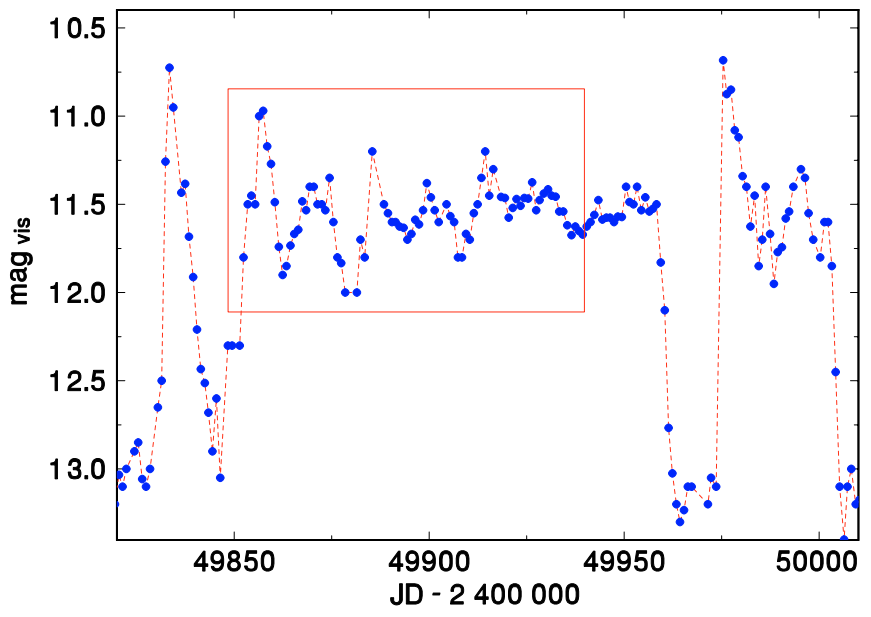

Fig. 11. The visual light curve of the dwarf nova Z Cam (one-day means of the observations from the AFOEV database). The light curve outlined by the box represents the interval in which $\dot{m}$ is nearly critical. (This figure is available in color in electronic form.)

The decays of the outbursts in 4U 1915-05 display a broad range of slopes. The slow average $\tau_{\mathrm{D}} \approx 0.2 \mathrm{ct} / \mathrm{s} /$ day $(D \approx$ 8.2 days) may be related to the viscous time of the disk $t_{\mathrm{vis}}$. Setting $\dot{m} \approx 2.5 \times 10^{-10}$ and $10^{-9} M_{\odot} \mathrm{yr}^{-1}$ and making use of Eq. (4) of Esin et al. (2000) lead to $t_{\mathrm{vis}} \approx 18$ and 12 days, respectively, but only if the viscosity parameter $\alpha$ is as high as 0.5 . The fastest decay $\left(\tau_{\mathrm{D}} \approx 0.35 \mathrm{ct} / \mathrm{s} /\right.$ day, $D \approx 4.6$ days $)$ would require $\alpha>1$. For comparison, the dwarf nova outbursts of SU UMa stars that have only slightly longer $P_{\text {orb }}$ (typically 81-120 min, e.g. Warner 1995) finish much faster than those of 4U 1915-05. The final decays of the outbursts of SU UMa stars are controlled by the cooling front which moves on the thermal timescale of the disk. However, the cooling front controlled by the irradiation by the NS in 4U 1915-05 (following the model by King \& Ritter 1998) gives rise to a much slower decay - this discrepancy can thus be solved. We note that the scatter of the AS M data for 4U 1915-05 is too large to resolve between the exponential decay, appropriate for the decay of a disk which is kept in the ionized state out to its outer rim by irradiation, and the linear decay, when the outer disk region remains below the hydrogen ionization temperature. The asymmetry of the disk and the spiral arms can offer an explanation for the outburst to outburst variation of the decay rate. The decay rate of most events appears to occur on the viscous timescale, with some contribution of the thermal timescale in some outbursts.

In summary, we argue that the observed long-term activity of 4U 1915-05 can be explained if its disk oscillates between thermally stable and unstable state. A very similar behaviour occurs in Z Cam-type dwarf novae in the intervals when their $\dot{m}$ approaches $\dot{m}_{\text {crit }}$ (Buat-Ménard et al. 2001). Figure 11 is an example: the amplitude of the outbursts largely diminishes and also the brightness at minimum increases considerably because the mass accretion onto the white dwarf still proceeds. This can help explain the substantially smaller amplitude of the intensity variations of 4U 1915-05 in both the X-ray and optical region in comparison with SXTs in the framework of TI. 
In the light of the findings presented above, it is tempting to link the variations of $P$ in 4U 1915-05 to those of dwarf novae (e.g. Šimon 2000, 2004a) and SXTs (Šimon 2004c). For example, GRS $1747-312$ displayed a mean $T_{\mathrm{C}} \approx 131$ days modulated with $P \approx 4.3$ years between the years 1996-2003 (Šimon 2004c).

Acknowledgements. This research has made use of NASA's Astrophysics Data System Abstract Service, and the observations provided by the $A S M / R X T E$ team. The optical observations of $\mathrm{Z} \mathrm{Cam}$, from the AFOEV database, Strasbourg, France, were utilized. The support by the project ESA PRODEX INTEGRAL 14527 is acknowledged. I made use of the code developed by G. Foster and available at http://www.aavso.org/data/software/wwz.shtml. I thank Dr. Hudec for reading the manuscript and for his comments. I am indebted to Dr. Harmanec for providing me with the program HEC13.

\section{References}

Barret, D., Grindlay, J. E., Strickman, M., \& Vedrenne, G. 1996, A\&AS, 120, 269

Becker, R. H., Smith, B. W., Swank, J. H., et al. 1977, ApJ, 216, L101

Bloser, P. F., Grindlay, J. E., Barret, D., \& Boirin, L. 2000, ApJ, 542, 989

Boyd, P. T., \& Smale, A. P. 2004, ApJ, 612, 1006

Buat-Ménard, V., Hameury, J.-M., \& Lasota, J.-P. 2001, A\&A, 369, 925

Callanan, P. J., Grindlay, J. E., \& Cool, A. M. 1995, PASJ, 47, 153

Chevalier, C., \& Ilovaisky, S. A. 1995, A\&A, 297, 103

Chou, Y., Grindlay, J. E., \& Bloser, P. F. 2001, ApJ, 549, 1135

Chou, Y., \& Grindlay, J. E. 2001, ApJ, 563, 934

Esin, A. A., Lasota, J.-P., \& Hynes, R. I. 2000, A\&A, 354, 987

Foster, G. 1996, AJ, 112, 1709,

Gerend, D., \& Boynton, P. E. 1976, ApJ, 209, 562

Grindlay, J. E., Bailyn, C. D., Cohn, H., et al. 1988, ApJ, 334, L25

Homer, L., Charles, P. A., Hakala, P., et al. 2001, MNRAS, 322, 827

King, A. R., \& Ritter, H. 1998, MNRAS, 293, L42

Leahy, D. A. 2002, A\&A, 391, 219

Levine, A. M., Bradt, H., Cui, W., et al. 1996, ApJ, 469, L33

Lomb, N. R. 1976, Astrophys. Space Sci., 39, 447

Mazeh, T., \& Shaham, J. 1979, A\&A, 77, 145
McGowan, K. E., Priedhorsky, W. C., \& Trudolyubov, S. P. 2004, ApJ, 601,1100

Menou, K., Perna, R., \& Hernquist, L. 2002, ApJ, 564, L81

Nelson, L. A., Rappaport, S. A., \& Joss, P. C. 1986, ApJ, 304, 231

Predehl, P., \& Schmitt, J. H. M. M. 1995, A\&A, 293, 889

Priedhorsky, W. C., \& Terrell, J. 1984, ApJ, 280, 661

Pylyser, E. H. P., \& Savonije, G. J. 1988, A\&A, 191, 57

Pylyser, E. H. P., \& Savonije, G. J. 1989, A\&A, 208, 52

Retter, A., Chou, Y., Bedding, T. R., \& Naylor, T. 2002, MNRAS, 330 , L37

Scargle, J. D. 1982, ApJ, 263, 835

Schreier, E., Giacconi, R., Gursky, H., et al. 1972, ApJ, 178, L71

Smale, A. P., \& Lochner, J. C. 1992, ApJ, 395, 582

Stella, L., White, N. E., \& Priedhorsky, W. 1987, ApJ, 315, L49

Stellingwerf, R. F. 1978, ApJ, 224, 953

Šimon, V. 2000, A\&A, 354, 103

Šimon, V. 2002, A\&A, 381, 151

Šimon, V. 2003, A\&A, 405, 199

Šimon, V. 2004a, Balt. Ast., 13, 101

Šimon, V. 2004b, Nucl. Phys. B, Proc. Suppl., 132, 656

Šimon, V. 2004c, Proc. of the 5th INTEGRAL Workshop: The INTEGRAL Universe, SP-552, ed. V. Schonfelder, G. Lichti, \& C. Winkler (Noordwijk, The Netherlands: ESA Publications Division, ESTEC), 399

Šimon, V. 2004d, A\&A, 418, 617

Truss, M. R., Murray, J. R., \& Wynn, G. A. 2001, MNRAS, 324, L1

Truss, M. R., Wynn, G. A., Murray, J. R., \& King, A. R. 2002, MNRAS, 337, 1329

Tuohy, I. R., \& Rapley, C. G. 1975, ApJ, 198, L69

van Paradijs, J., van der Klis, M., \& Pedersen, H. 1988, A\&AS, 76, 185

Vondrák, J. 1969, Bull. Astron. Inst. Czechosl., 20, 349

Vondrák, J. 1977, Bull. Astron. Inst. Czechosl., 28, 84

Walter, F. M., Mason, K. O., Clarke, J. T., et al. 1982, ApJ, 253, L67

Warner, B. 1995, Cataclysmic Variable Stars (Cambridge: Cambridge Univ. Press)

Wijers, R. A. M. J., \& Pringle, J. E. 1999, MNRAS, 308, 207

Wilms, J., Nowak, M. A., Pottschmidt, K., et al. 2001, MNRAS, 320, 327

Whittaker, E., \& Robinson, G. 1946, The Calculus of Observations, (London: Blackie \& Son Ltd), 303

Yoshida, K. 1993, Ph.D. Thesis, Univ. Tokyo 